\title{
Transverse Momentum Differential Global Analysis of Heavy-Ion Collisions
}

\author{
Govert Nijs $\odot,{ }^{1,2, *}$ Wilke van der Schee, ${ }^{3, \dagger}$ Umut Gürsoy, ${ }^{2, \ddagger}$ and Raimond Snellings ${ }^{4,5, \S}$ \\ ${ }^{1}$ Center for Theoretical Physics, Massachusetts Institute of Technology, Cambridge, Massachusetts 02139, USA \\ ${ }^{2}$ Institute for Theoretical Physics and Center for Extreme Matter and Emergent Phenomena, \\ Utrecht University, 3584 CC Utrecht, Netherlands \\ ${ }^{3}$ Theoretical Physics Department, CERN, CH-1211 Genève 23, Switzerland \\ ${ }^{4}$ Institute for Gravitational and Subatomic Physics (GRASP), Utrecht University, 3584 CC Utrecht, Netherlands \\ ${ }^{5}$ Nikhef, 1098 XG Amsterdam, Netherlands
}

(Received 5 November 2020; revised 26 February 2021; accepted 12 April 2021; published 20 May 2021)

\begin{abstract}
The understanding of heavy ion collisions and its quark-gluon plasma (QGP) formation requires a complicated interplay of rich physics in a wealth of experimental data. In this work we compare for identified particles the transverse momentum dependence of both the yields and the anisotropic flow coefficients for both $\mathrm{PbPb}$ and $\mathrm{pPb}$ collisions. We do this in a global model fit including a free streaming prehydrodynamic phase with variable velocity $v_{\mathrm{fs}}$, thereby widening the scope of initial conditions. During the hydrodynamic phase we vary three second order transport coefficients. The free streaming velocity has a preference slightly below the speed of light. In this extended model the QGP bulk viscosity is small and even consistent with zero.
\end{abstract}

DOI: 10.1103/PhysRevLett.126.202301

Introduction.-The quark-gluon plasma (QGP) is a state of deconfined matter of quarks and gluons whose existence at high energy density is predicted by quantum chromodynamics. Heavy ion collisions (HIC) at RHIC and LHC have led to a wealth of data from which the formation of this quark-gluon plasma can be inferred $[1,2]$. This existence can be deduced by having a model of initial conditions directly after the collision, a hydrodynamic phase with certain transport properties, and, lastly, a hadronic phase of which the results can be compared to experimental results. Even though robust conclusions on the existence of a QGP can be reached from qualitative features of the data, such as the anisotropy of the low transverse momentum particles or the quenching of high momentum partons, for a quantitative understanding of fundamental properties such as, e.g., the shear and bulk viscosity, it is paramount to have a careful understanding of all parameters involved in all initial, hydrodynamic, and hadronic phases.

Early studies performing such a comprehensive analysis where all parameters in all stages can be varied simultaneously include Refs. [3-9]. This is done in similarity to modeling in cosmology [10], where cosmological parameters have to be inferred from the cosmic microwave background as well as large scale structure analysis. Full simulations themselves are computationally expensive, and

Published by the American Physical Society under the terms of the Creative Commons Attribution 4.0 International license. Further distribution of this work must maintain attribution to the author(s) and the published article's title, journal citation, and DOI. hence an emulator trained on a few carefully selected design points is used to evaluate the likelihood of parameters using a Markov chain Monte Carlo $(\mathrm{mcmc})$. This, together with typically flat prior probability distributions, leads to final (Bayesian) posterior distributions for the chosen parameters.

For a precision study of QGP properties, the scope of the full model is important, as artificially restricting, e.g., the range of initial conditions may pose unphysical restrictions on hydrodynamic transport. In this Letter we will present the widest set of initial conditions studied to date combined with hydrodynamics with varying second order transport coefficients, containing a total of 21 varying parameters (boldface in this Letter). Most importantly, we perform a global analysis including experimental data with transverse momentum dependence as well as particle identification for spectra and anisotropic flow coefficients for $\mathrm{PbPb}$ collisions at 2.76 and $5.02 \mathrm{TeV}$, together with identified spectra for $p \mathrm{~Pb}$ collisions at $5.02 \mathrm{TeV}$.

Model.-For the initial conditions we use the TRENTo model parametrization $[11,12]$. In this model nucleons of Gaussian width w are positioned by a fluctuating Glauber model separated by a distance of at least $\mathbf{d}_{\min }$, and all nucleons consist of $\mathbf{n}_{c}$ randomly placed constituents having a Gaussian width of $v_{\min }+\chi_{\text {struct }}\left(\mathbf{w}-v_{\min }\right)$, with $v_{\min }=$ $0.2 \mathrm{fm}$ Nucleons are wounded depending on their overlap such that the cross section matches the proton-proton result. Constituents of wounded nucleons contribute to the left and right thickness functions $\mathcal{T}_{A}$ and $\mathcal{T}_{B}$ with norm $\mathbf{N} \gamma / \mathbf{n}_{c}$, where $\gamma$ fluctuates according to a gamma distribution of width $\boldsymbol{\sigma}_{\text {fluct }} \sqrt{\mathbf{n}_{c}}$. These functions are finally combined to a 
final parton density by $\mathcal{T}=\left(\frac{1}{2} \mathcal{T}_{A}^{\mathbf{p}}+\frac{1}{2} \mathcal{T}_{B}^{\mathbf{p}}\right)^{1 / \mathbf{p}}$ with $\mathbf{p}$ a free parameter.

The prehydrodynamic evolution consists of a freestreaming phase lasting for a time $\tau_{\mathrm{fs}}$, with the new feature of introducing an effective velocity $\mathbf{v}_{\mathbf{f s}}$ (see also the discussion).

For the hydrodynamic evolution, we solve the conservation equations for the stress-energy tensor, with the stress-energy tensor given by the hydrodynamic constitutive relation: $T^{\mu \nu}=\rho u^{\mu} u^{\nu}-(P+\Pi) \Delta^{\mu \nu}+\pi^{\mu \nu}$, where $\Delta^{\mu \nu}=g^{\mu \nu}-u^{\mu} u^{\nu}$, and we use the mostly minus convention for the metric. Here $\rho, P, \Pi$, and $\pi^{\mu \nu}$ are the energy density, pressure, bulk viscous pressure, and the traceless transverse shear stress, respectively. The equations of motion for the bulk pressure $\Pi$ and the shear stress $\pi^{\mu \nu}$ are given by the 14moment approximation [13], where we keep only the transport coefficients used in [7]:

$$
\begin{aligned}
D \Pi= & -\frac{1}{\tau_{\Pi}}\left[\Pi+\zeta \nabla \cdot u+\delta_{\Pi \Pi} \nabla \cdot u \Pi-\lambda_{\Pi \pi} \pi^{\mu \nu} \sigma_{\mu \nu}\right], \\
\Delta_{\alpha}^{\mu} \Delta_{\beta}^{\nu} D \pi^{\alpha \beta}= & -\frac{1}{\tau_{\pi}}\left[\pi^{\mu \nu}-2 \eta \sigma^{\mu \nu}+\delta_{\pi \pi} \pi^{\mu \nu} \nabla \cdot u\right. \\
& \left.-\phi_{7} \pi_{\alpha}^{\langle\mu} \pi^{\nu\rangle \alpha}+\tau_{\pi \pi} \pi_{\alpha}^{\langle\mu} \sigma^{\nu\rangle \alpha}-\lambda_{\pi \Pi} \Pi \sigma^{\mu \nu}\right] .
\end{aligned}
$$

The pressure is given in terms of the energy density by the hybrid HotQCD/HRG equation of state [14-16]. We parametrize the first order transport coefficients $\eta$ and $\zeta$ in terms of the dimensionless ratios $\eta / s$ and $\zeta / s$ In particular, $\eta / s=a+b\left(T-T_{c}\right)\left(T / T_{c}\right)^{c}$, with a minimal value $a=$ $(\boldsymbol{\eta} / \mathbf{s})_{\min }$ at $T_{c}=154 \mathrm{MeV}$, a slope $b=(\boldsymbol{\eta} / \mathbf{s})_{\text {slope }}$ and a curvature $c=(\boldsymbol{\eta} / \mathbf{s})_{\text {crv }}$. The bulk viscosity $\zeta / s$ is described by an unnormalized Cauchy distribution with height $(\zeta / \mathbf{s})_{\max }$, width $(\zeta / \mathbf{s})_{\text {width }}$, and peak temperature $(\zeta / \mathbf{s})_{T_{0}}$. The second order transport coefficients $\tau_{\Pi}, \delta_{\Pi \Pi}, \lambda_{\Pi \pi}, \tau_{\pi}$, $\delta_{\pi \pi}, \phi_{7}, \tau_{\pi \pi}$, and $\lambda_{\pi \Pi}$ are also given in terms of dimensionless ratios. Of these, we fix

$$
\begin{aligned}
\frac{\delta_{\Pi \Pi}}{\tau_{\Pi}}=\frac{2}{3}, & \frac{\lambda_{\Pi \pi}}{\tau_{\Pi} \delta}=\frac{8}{5}, & \frac{\delta_{\pi \pi}}{\tau_{\pi}}=\frac{4}{3}, \\
\phi_{7} P=\frac{9}{70}, & \frac{\lambda_{\pi \Pi}}{\tau_{\pi}}=\frac{6}{5} &
\end{aligned}
$$

to the values from kinetic theory [13], with $\delta=1 / 3-c_{s}^{2}$, while we vary the shear and bulk relaxation times $\tau_{\pi}$ and $\tau_{\Pi}$ as well as one other second order coefficient $\tau_{\pi \pi}$. We vary these according to the ratios

$$
\frac{\tau_{\Pi} \mathbf{S} \mathbf{T} \delta^{2}}{\zeta}, \quad \frac{\tau_{\pi} \mathbf{s} \mathbf{T}}{\eta} \text { and } \frac{\tau_{\pi \pi}}{\tau_{\pi}}
$$

Finally, the hydrodynamic fluid undergoes particlization at a temperature $\mathbf{T}_{\text {switch }}$, whereby viscous contributions as well as resonances are included according to the algorithms presented in Refs. [16,17]. These hadrons are then evolved using the SMASH hadronic cascade code [18-20].

Experimental data.-To compare our model to experiment we start with the dataset used in Ref. [6]: $\mathrm{PbPb}$ charged particle multiplicity $d N_{\mathrm{ch}} / d \eta$ at 2.76 [21] and $5.02 \mathrm{TeV}$ [22], transverse energy $d E_{T} / d \eta$ at $2.76 \mathrm{TeV}$ [23], identified yields $d N / d y$ and mean $p_{T}$ for pions, kaons, and protons at $2.76 \mathrm{TeV}$ [24], integrated anisotropic flow $v_{n}\{k\}$ for both 2.76 and $5.02 \mathrm{TeV}$ [25], and $p_{T}$ fluctuations [26] at $2.76 \mathrm{TeV}$. On top of this we added identified transverse momentum spectra using six coarse-grained $p_{T}$ bins separated at $(0.5,0.75,1.0,1.4,1.8,2.2,3.0) \mathrm{GeV}$ both for $\mathrm{PbPb}$ at 2.76 [24] and $p \mathrm{~Pb}$ at $5.02 \mathrm{TeV}$ [27], anisotropic identified flow coefficients using the same $p_{T}$ bins (statistics allowing) at 2.76 [28] and 5.02 TeV [29]. As in Ref. [30] we use $\tilde{v}_{n}\{k\}$ anisotropic flow coefficients for $p \mathrm{~Pb}$ at $5.02 \mathrm{TeV}[31,32]$ as well as mean $p_{T}$ for pions, kaons, and protons at $5.02 \mathrm{TeV}$ [33]. All of these use representative centrality classes, whereby we also specifically included high multiplicity $p \mathrm{~Pb}$ classes for its anisotropic flow coefficients, giving a total of 418 and 96 datapoints for $\mathrm{PbPb}$ and $p \mathrm{~Pb}$ collisions, respectively.

Posterior distribution.-In order to estimate the likelihood of all 21 parameters (bold in the model) we used Trajectum [34] to simulate the full $\mathrm{PbPb}(p \mathrm{~Pb})$ model at 1000 (2000) design points located on a Latin Hypercube in the parameter space using $6 \mathrm{k}(40 \mathrm{k})$ events per design point (the parameter ranges can be found in the posterior distributions later) [35]. For each system we apply a transformation to 25 principal components (PCs), for which we train Gaussian emulators $[16,30,36]$. Crucially, the emulator also estimates its own uncertainty (which we validated) and through the principle component analysis this includes correlations among the datapoints. Full details as well as emulator results can be found in our companion paper [37].

Using either $\mathrm{PbPb}$ only or both $\mathrm{PbPb}$ and $p \mathrm{~Pb}$ emulators we ran a Markov Chain Monte Carlo (mcmc) employing the EMCEE2.2 code [16,30,38], using 600 walkers for approximately $15 \mathrm{k}$ steps. This led to the converged posterior distributions in Fig. 1, shown with (solid) and without (dashed) the $\mathrm{pPb}$ data. Figure 2 shows results from 100 random samples of the posterior distribution for a representative selection of our datapoints. In general these compare well, even for $p_{T}$-differentiated identified $v_{n}\{2\}$ distributions for both central and peripheral collisions.

For $p \mathrm{~Pb}$ the posterior distributions are significantly wider than the experimental uncertainties, since even for 2000 design points the model is sufficiently complicated that a significant emulator uncertainty remains. It is for this reason that including $p \mathrm{~Pb}$ for the posterior (blue solid versus green dashed in Fig. 1) does not change the probabilities as much as perhaps expected, though for parameters especially sensitive to small and short-lived 

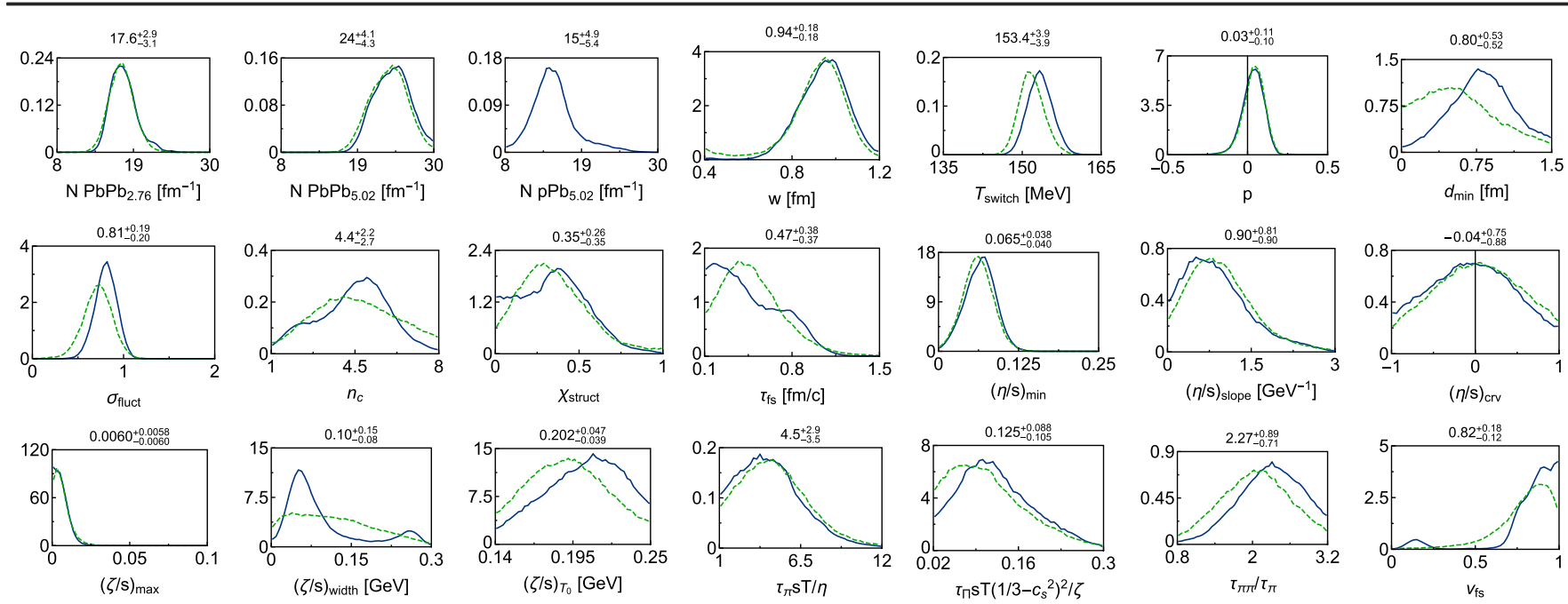

FIG. 1. Posterior distributions for all model parameters fitted to $\mathrm{PbPb}$ and $p \mathrm{~Pb}$ (solid) or $\mathrm{PbPb}$ only (dashed, not applicable to $p \mathrm{~Pb}$ norm) data. Values indicate the expectation values with the $90 \%$ highest posterior density credible interval.

systems better constraints are obtained $\left(\mathbf{n}_{c}, \boldsymbol{\tau}_{\mathrm{fs}}, \mathbf{w}, \mathbf{d}_{\min }\right.$, and $\left.\sigma_{\text {fluct }}\right)$.

Perhaps the most striking feature in Fig. 1 is that the posterior for the maximum of $\zeta / s$ peaks at zero. This is in contrast to previous work $[6,7,39]$ that prefers a positive bulk viscosity in order to reduce the mean $p_{T}$. A larger bulk viscosity, however, makes it hard to describe the $p_{T}$ identified spectra [Fig. 2 (middle,top)].

Given the scope of our 21 parameter model it is perhaps not surprising that constraints on the parameters and in particular the second order transport coefficients are not that strong. We do however see interesting correlations, as shown in Fig. 3. As expected $(\boldsymbol{\eta} / \mathbf{s})_{\min }$ and $(\boldsymbol{\eta} / \mathbf{s})_{\text {slope }}$ are negatively correlated. Perhaps the most interesting correlation is between $\boldsymbol{\tau}_{\mathrm{fs}}$ and $\boldsymbol{\tau}_{\pi} \mathbf{s T} / \boldsymbol{\eta}$ : it is possible to have a rather long free-streaming time, but only if $\tau_{\pi}$ is relatively small. This correlation indeed guarantees the quick applicability of (viscous) hydrodynamics. In the pre-equilibrium stage, the larger $\boldsymbol{\tau}_{\mathrm{fs}}$ the larger the deviation from hydrodynamics; see also Refs. [37,40]. On the other hand, a
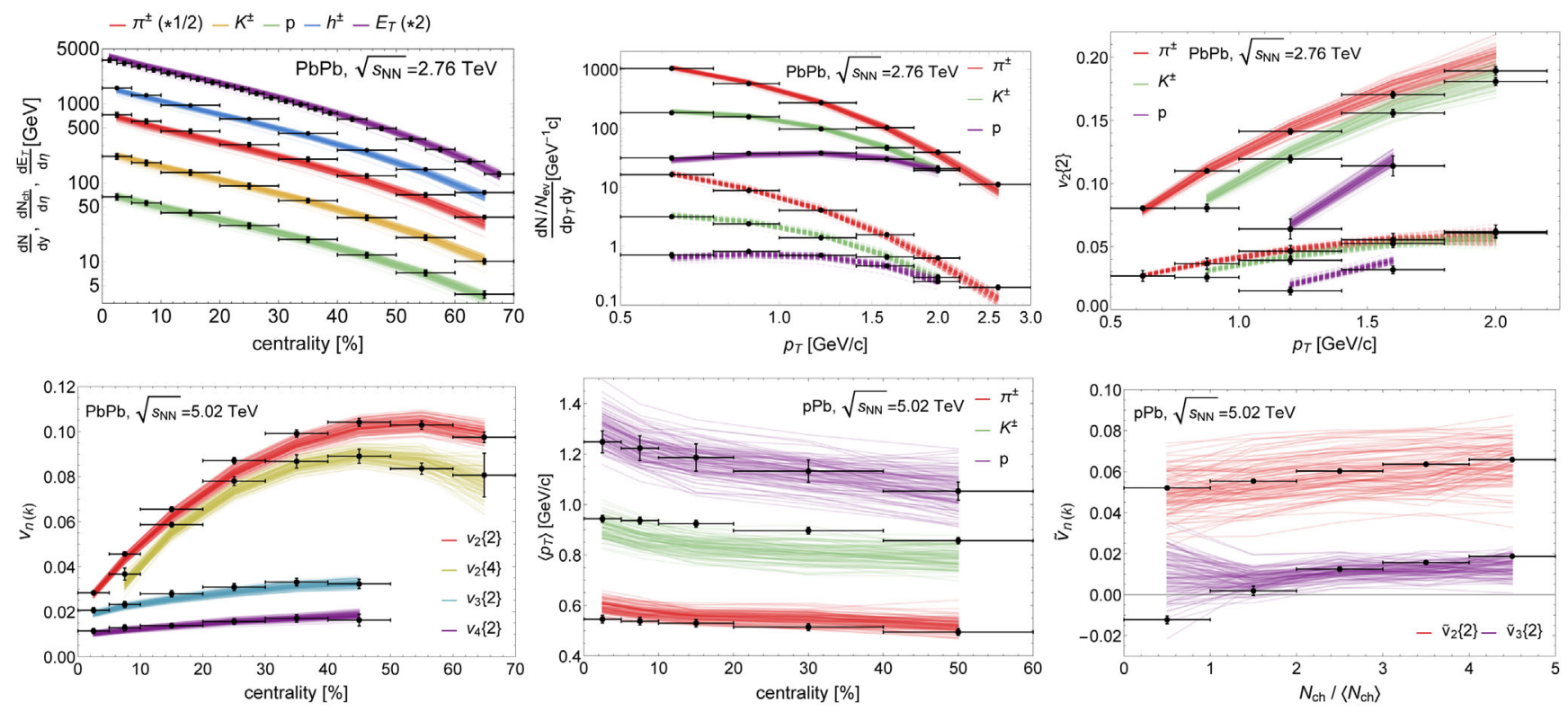

FIG. 2. A selection of the experimental data used together with 100 samples drawn from Fig. 1. (top) Multiplicities and transverse energy versus centrality (left); $p_{T}$ spectra for pions, kaons, and protons for $0 \%-5 \%$ (solid) and $40 \%-50 \%$ (dashed, *0.1) centrality classes (middle); and $v_{2}\{2\}$ versus $p_{T}$ for 0\%-5\% (dashed) and 20\%-30\% (solid) (right). (bottom) $v_{n}\{k\}$ versus centrality for PbPb collisions at top LHC energy (left), mean transverse momenta for $p \mathrm{~Pb}$ collisions versus centrality (middle), and $\tilde{v}_{n}\{k\}$ for $p \mathrm{~Pb}$ collisions depending on multiplicity class (right). 

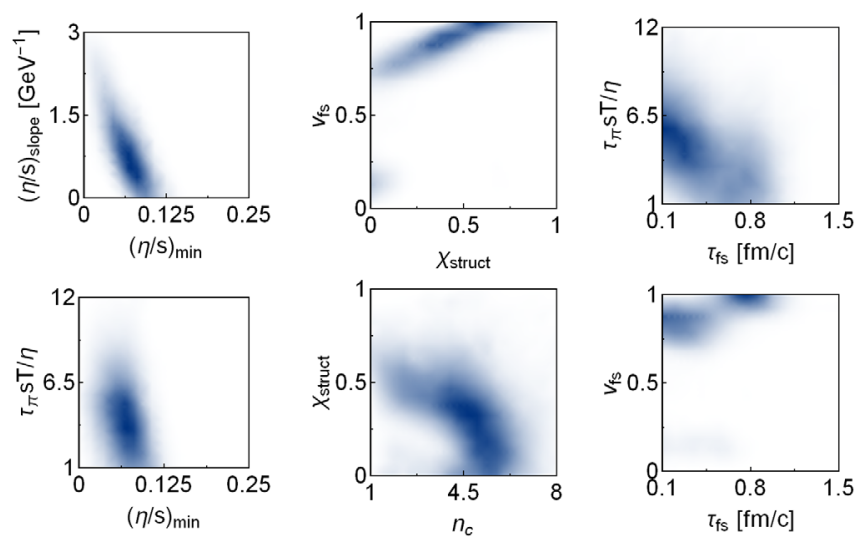

FIG. 3. We highlight a few interesting or strong correlations among the posterior distributions shown in Fig. 1.

shorter shear relaxation time dampens large deviations from viscous hydrodynamics more quickly. In this way, we can interpret the joint constraint on $\boldsymbol{\tau}_{\pi} \mathbf{s} \mathbf{T} / \boldsymbol{\eta}$ and $\boldsymbol{\tau}_{\mathrm{fs}}$ from the posterior distribution as a preference for the fluid to quickly hydrodynamize [41]. Another strong negative correlation is between $\mathbf{v}_{\mathrm{fs}}$ and $\chi_{\text {struct }}$, and indeed for $\mathbf{v}_{\mathrm{fs}}=1$ our $\chi_{\text {struct }}$ distribution is in agreement with Ref. [12]. This highlights the importance of gaining a better understanding of the initial stages of the collision. We also find that $\mathbf{n}_{c}$ and $\chi_{\text {struct }}$ are negatively correlated.

We obtain tight constraints on our prehydrodynamic flow parameter $\mathbf{v}_{\mathrm{fs}}$. Indeed the preferred value is close to unity, with perhaps an unlikely option of a small velocity combined with a tiny $\chi_{\text {struct }}$. From Fig. 3 we see that either there is a short $\boldsymbol{\tau}_{\mathrm{fs}}$ with $\mathbf{v}_{\mathrm{fs}} \approx 0.85$, or a longer $\boldsymbol{\tau}_{\mathrm{fs}}$ with $\mathbf{v}_{\mathrm{fs}}=1$. The first option is consistent with an equation of state that is slightly below the conformal limit; indeed at $T=0.4 \mathrm{GeV}$ the pressure equals $85 \%$ of its conformal value $[14,16]$. In this scenario the fluid is initialized with a bulk pressure close to its ideal hydrodynamic value at an early time (see also Ref. [37]). In the second scenario the fluid will start further from equilibrium, but here the influence of $\mathbf{v}_{\mathrm{fs}}$ on the initial geometry is dominant, as is also clear from the correlation with $\chi_{\text {struct }}$.

Discussion.-From the posterior distributions it is possible to obtain the $90 \%$ confidence limit of the viscosities, as shown in Fig. 4. For lower temperatures the viscosity is consistent with the canonical string theory value of $1 / 4 \pi$ [42] (note also that stringy models exist with a lower viscosity [43]). There is a clear tendency for $\eta / s$ to increase, as expected from the running of the coupling constant. As already shown the bulk viscosity is found to be small, which is consistent with an approximately conformal theory. This contrasts with the prevailing view that a finite bulk viscosity $[6,7,39]$ is needed in order to simultaneously fit the mean transverse momenta and anisotropic flow. We also obtain mild constraints on $\boldsymbol{\tau}_{\pi} \mathbf{s} \mathbf{T} / \boldsymbol{\eta} \lesssim 7$ and $\boldsymbol{\tau}_{\pi \pi} / \boldsymbol{\tau}_{\pi} \gtrsim 1.5$. The value for $\boldsymbol{\tau}_{\pi} \mathbf{s} \mathbf{T} / \boldsymbol{\eta}$ compares well with the holographic $[4-\log (4) \approx 2.61$,
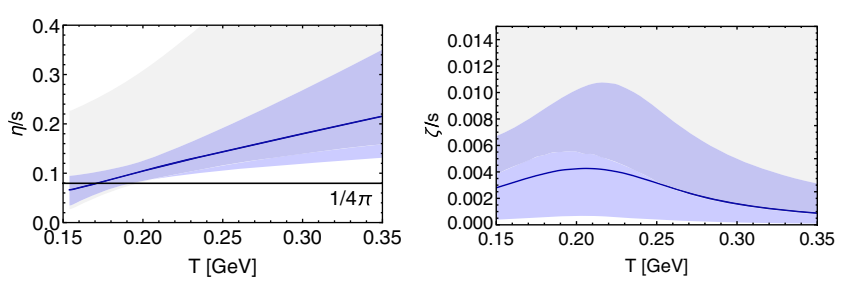

FIG. 4. Posterior distributions for the specific shear and bulk viscosities versus temperature, together with their mean and $90 \%$ confidence band (blue). The $90 \%$ confidence bands for the prior distribution is shown in gray (extending till 0.08 for $\zeta / s$, not shown).

[44] ] and weak coupling (5, [13]) results. The $\boldsymbol{\tau}_{\pi \pi} / \boldsymbol{\tau}_{\pi}$ value is consistent with the weak coupling result $(10 / 7 \approx 1.43$, [45]) and agrees well with the holographic result $(88 / 35[2-\log (2)] \approx 1.92,[46])$.

We performed several extra mcmc analyses in order to better understand our small bulk viscosity, shown in Fig. 5 . There we varied the observables used (all versus those in Ref. [6] labeled Duke) and similarly our varying parameters and lastly including $p \mathrm{~Pb}$ or not. Setting both observables, parameters and systems to those in Ref. [6] reproduces their bulk viscosity. Including $p \mathrm{~Pb}$, more parameters and, most importantly, including our $p_{T}$-differential observables all reduce the bulk viscosity, explaining the result in Fig. 4. The fact that setting our parameters to the ones used in Ref. [6] reproduces the small bulk viscosity in particular
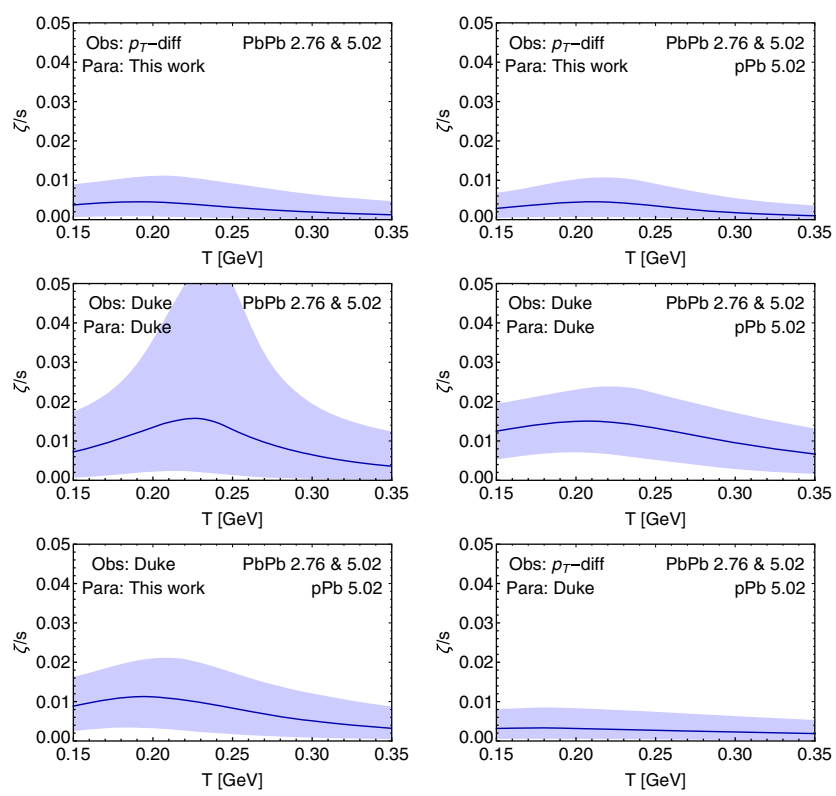

FIG. 5. We show posteriors for $(\boldsymbol{\zeta} / \mathbf{s})_{\max }$ as in Fig. 4 in variations using the model presented, or limiting to a subset with fewer observables (as in Ref. [6], labeled Duke), fewer parameters (as in Ref. [6], labeled Duke), or without the $p \mathrm{~Pb}$ system. The new $p_{T}$-differential observables are the most significant addition that led to the small bulk viscosity in Fig. 4. 

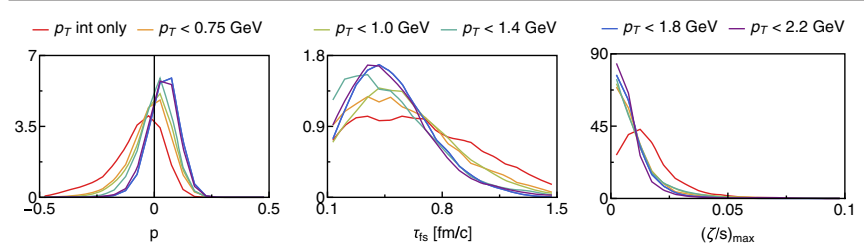

FIG. 6. We vary the maximum $p_{T}$ used in the $p_{T}$-differentiated analysis for three representative parameters. Clearly for the bulk viscosity the highest gain in precision is present by including low $p_{T}$ particles, whereas the Trento parameter and free streaming time benefit more gradually from higher $p_{T}$ data.

implies that setting $v_{\mathrm{fs}}=1$ does not lead to a larger bulk viscosity, but instead gives a smoother subnucleonic structure as is clear from the $v_{\mathrm{fs}}-\chi_{\text {struct }}$ correlation in Fig. 3.

A crucial question on our analysis is how much information is gained by the respective $p_{T}$ bins and how sensitive our results are to the observables at high $p_{T}$. This is particularly important, since our viscous freeze-out prescription $[16,17]$ has significant systematic uncertainty that is more important at high $p_{T}$ (see also Ref. [6]). It is hence important to verify that our main conclusions are not sensitive to our particular freeze-out prescription, and indeed we see in Fig. 6 that the bulk viscosity is almost entirely determined by the low $p_{T}$ bins. For other observables such as the free streaming time, a more gradual increase in precision is observed, but none of our posteriors depend sensitively on our highest $p_{T}$ bin between 2.2-3.0 GeV.

It is still debated whether matter formed in $p \mathrm{~Pb}$ collisions can be described by hydrodynamics [47-49]. Indeed, one of our main motivations of this study was to shed light on this question, by computing posterior probabilities with and without $p \mathrm{~Pb}$ collisions. If our framework manages to fit $p \mathrm{~Pb}$ well this gives further evidence for a hydrodynamic picture. In general, we agree well with $p \mathrm{~Pb}$ observables, but the mean kaon $\left\langle p_{T}\right\rangle$ seems to deviate significantly (see also Ref. [37]). This can either imply a deviation from the hydrodynamic picture, but given that our $p \mathrm{~Pb}$ observables are much harder to emulate, it could also indicate a more advanced analysis within hydrodynamics or a more advanced initial stage is needed.

Our model can be improved in two directions. First, our initial state, prehydrodynamic phase and particlization are not based on a microscopic theory and in particular the transition to hydrodynamics is not smooth [50]. It would be interesting to investigate this point by including a more physically motivated initial stage. Second, we were only able to use data that can be reliably estimated using about $6 \mathrm{k}$ events, whereas much more sophisticated data is available. Our data include the widest set available for a global analysis so far, but nevertheless only roughly 20 principal components are nontrivial. This is much more than in, e.g., Refs. $[9,12,16]$ where up to 8 PCs contain over $99 \%$ of the nontrivial information. Nevertheless the question remains whether it is possible to estimate so many parameters with relatively limited experimental data (see also Ref. [51] where it is found that using only the limited dataset it is difficult to obtain much stronger constraints than the given prior range). In the future it will hence be important to incorporate more nontrivial data, perhaps using some approximations to reduce computation time (see also Refs. [37,52]).

We are grateful to Jonah Bernhard and Scott Moreland for making their codes public together with an excellent documentation. We thank Steffen Bass, Aleksas Mazeliauskas, Ben Meiring, and Urs Wiedemann for discussions. G. N. is supported by the U.S. Department of Energy, Office of Science, Office of Nuclear Physics under grant Contract No. DE-SC0011090.

*govert@mit.edu

wilke.van.der.schee@cern.ch

*u.gursoy@uu.nl

${ }^{\S}$ raimond.snellings@nikhef.nl

[1] U. Heinz and R. Snellings, Annu. Rev. Nucl. Part. Sci. 63, 123 (2013).

[2] W. Busza, K. Rajagopal, and W. van der Schee, Annu. Rev. Nucl. Part. Sci. 68, 339 (2018).

[3] J. Novak, K. Novak, S. Pratt, J. Vredevoogd, C. ColemanSmith, and R. L. Wolpert, Phys. Rev. C 89, 034917 (2014).

[4] S. Pratt, E. Sangaline, P. Sorensen, and H. Wang, Phys. Rev. Lett. 114, 202301 (2015).

[5] E. Sangaline and S. Pratt, Phys. Rev. C 93, 024908 (2016).

[6] J. E. Bernhard, J. S. Moreland, S. A. Bass, J. Liu, and U. Heinz, Phys. Rev. C 94, 024907 (2016).

[7] J. E. Bernhard, J. S. Moreland, and S. A. Bass, Nat. Phys. 15, 1113 (2019).

[8] D. Devetak, A. Dubla, S. Floerchinger, E. Grossi, S. Masciocchi, A. Mazeliauskas, and I. Selyuzhenkov, J. High Energy Phys. 06 (2020) 044.

[9] J. Auvinen, K. J. Eskola, P. Huovinen, H. Niemi, R. Paatelainen, and P. Petreczky, Phys. Rev. C 102, 044911 (2020).

[10] S. Habib, K. Heitmann, D. Higdon, C. Nakhleh, and B. Williams, Phys. Rev. D 76, 083503 (2007).

[11] J. S. Moreland, J. E. Bernhard, and S. A. Bass, Phys. Rev. C 92, 011901(R) (2015).

[12] J. S. Moreland, J. E. Bernhard, and S. A. Bass, Phys. Rev. C 101, 024911 (2020).

[13] G. S. Denicol, S. Jeon, and C. Gale, Phys. Rev. C 90, 024912 (2014).

[14] P. Huovinen and P. Petreczky, Nucl. Phys. A837, 26 (2010).

[15] A. Bazavov et al. (HotQCD Collaboration), Phys. Rev. D 90, 094503 (2014).

[16] J. E. Bernhard, Bayesian parameter estimation for relativistic heavy-ion collisions, Ph.D. thesis, Duke University, 2018.

[17] S. Pratt and G. Torrieri, Phys. Rev. C 82, 044901 (2010).

[18] J. Weil et al., Phys. Rev. C 94, 054905 (2016).

[19] D. Oliinychenko, V. Steinberg, J. Weil, M. Kretz, J. Staudenmaier, S. Ryu, A. Schfer, J. Rothermel, J. Mohs, 
F. Li, H. E. Petersen, L. Pang, D. Mitrovic, A. Goldschmidt, L. Geiger, J.-B. Rose, J. Hammelmann, and L. Prinz, smashtransport/smash: Smash-1.8, (2020).

[20] T. Sjostrand, S. Mrenna, and P. Z. Skands, Comput. Phys. Commun. 178, 852 (2008).

[21] K. Aamodt et al. (ALICE Collaboration), Phys. Rev. Lett. 106, 032301 (2011).

[22] J. Adam et al. (ALICE Collaboration), Phys. Rev. Lett. 116, 222302 (2016).

[23] J. Adam et al. (ALICE Collaboration), Phys. Rev. C 94, 034903 (2016).

[24] B. Abelev et al. (ALICE Collaboration), Phys. Rev. C 88, 044910 (2013).

[25] J. Adam et al. (ALICE Collaboration), Phys. Rev. Lett. 116, 132302 (2016).

[26] B. B. Abelev et al. (ALICE Collaboration), Eur. Phys. J. C 74, 3077 (2014).

[27] J. Adam et al. (ALICE Collaboration), Phys. Lett. B 760, 720 (2016).

[28] J. Adam et al. (ALICE Collaboration), J. High Energy Phys. 09 (2016) 164.

[29] S. Acharya et al. (ALICE Collaboration), J. High Energy Phys. 09 (2018) 006.

[30] J. S. Moreland, Initial conditions of bulk matter in ultrarelativistic nuclear collisions, Ph.D. thesis, Duke University, 2019.

[31] M. Aaboud et al. (ATLAS Collaboration), Eur. Phys. J. C 77, 428 (2017).

[32] Since in $p \mathrm{~Pb} v_{n}\{k\}$ can become imaginary we use $\tilde{v}_{n}\{k\} \equiv \operatorname{sgn}\left(v_{n}\{k\}^{k}\right)\left|v_{n}\{k\}\right|$.

[33] B. B. Abelev et al. (ALICE Collaboration), Phys. Lett. B 728, 25 (2014).

[34] Source code is available at https://sites.google.com/view/ govertnijs/trajectum.

[35] The computing time for Trajectum and SMASH are comparable, both taking roughly $10^{6}$ core hours.
[36] C. K. Williams and C. E. Rasmussen, Gaussian Processes for Machine Learning, Vol. 2 (MIT Press, Cambridge, MA, 2006).

[37] G. Nijs, W. van der Schee, U. Gürsoy, and R. Snellings, companion paper, Phys. Rev. C 103, 054909 (2021).

[38] D. Foreman-Mackey, D. W. Hogg, D. Lang, and J. Goodman, Publ. Astron. Soc. Pac. 125, 306 (2013).

[39] S. Ryu, J. F. Paquet, C. Shen, G. S. Denicol, B. Schenke, S. Jeon, and C. Gale, Phys. Rev. Lett. 115, 132301 (2015).

[40] J. Liu, C. Shen, and U. Heinz, Phys. Rev. C 91, 064906 (2015); 92, 049904(E) (2015).

[41] M. P. Heller, Acta Phys. Pol. B 47, 2581 (2016).

[42] G. Policastro, D. T. Son, and A. O. Starinets, Phys. Rev. Lett. 87, 081601 (2001).

[43] M. Brigante, H. Liu, R. C. Myers, S. Shenker, and S. Yaida, Phys. Rev. D 77, 126006 (2008).

[44] R. Baier, P. Romatschke, D. T. Son, A. O. Starinets, and M. A. Stephanov, J. High Energy Phys. 04 (2008) 100.

[45] E. Molnár, H. Niemi, G. S. Denicol, and D. H. Rischke, Phys. Rev. D 89, 074010 (2014).

[46] S. Bhattacharyya, R. Loganayagam, I. Mandal, S. Minwalla, and A. Sharma, J. High Energy Phys. 12 (2008) 116.

[47] R. D. Weller and P. Romatschke, Phys. Lett. B 774, 351 (2017).

[48] A. Kurkela, U. A. Wiedemann, and B. Wu, Eur. Phys. J. C 79, 965 (2019).

[49] J. L. Nagle and W. A. Zajc, Annu. Rev. Nucl. Part. Sci. 68, 211 (2018).

[50] W. van der Schee, P. Romatschke, and S. Pratt, Phys. Rev. Lett. 111, 222302 (2013).

[51] D. Everett et al. (JETSCAPE Collaboration), arXiv:2010 .03928.

[52] H. Niemi, K. J. Eskola, and R. Paatelainen, Phys. Rev. C 93, 024907 (2016). 